\title{
Stereodivergent-at-Metal Synthesis of [60]Fullerene Hybrids
}

\author{
Juan Marco-Martínez, Sara Vidal, Israel Fernández, Salvatore Filippone, * and Nazario Martín*
}

In memory of José Barluenga

\begin{abstract}
Chiral fullerene-metal hybrids with complete control over the four stereogenic centers, including the absolute configuration of the metal atom, have been synthesized for the first time. The stereochemistry of the four chiral centers formed during [60]fullerene functionalization is the result of both the chiral catalysts employed and the diastereoselective addition of the metal complexes used (iridium, rhodium, or ruthenium). DFT calculations underpin the observed configurational stability at the metal center, which does not undergo an epimerization process.
\end{abstract}

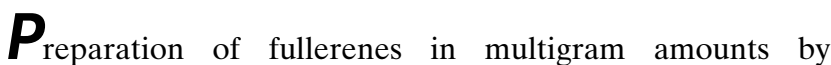
Krätschmer et al. in $1990^{[1]}$ permitted the study of the reactivity of the new carbon allotropes with transition-metal complexes, which subsequently arose as one of the main fields in fullerene science. ${ }^{[2]}$ To date, interest in fullerene-transitionmetal complexes has been renewed in the search for new applications and synergies arising from the complementary properties of transition metals and fullerenes. ${ }^{[3]}$ Transition metal involvement in organic transformations drives the investigation of new complexes with catalytic activity. In particular, preparative methods for chiral metal complexesspecifically chiral-at-metal complexes-are highly sought after in current organometallic chemistry because of their relevance in enantioselective metal-mediated processes. ${ }^{[4]}$

Despite the wealth of metal-fullerene hybrids reported to date, only a few are optically active..$^{[2,5]}$ To the best of our knowledge, only a single case presents a stereogenic metal center, ${ }^{[6]}$ while in all examples the chiral information stems from the stoichiometric use of chiral ligands. ${ }^{[7]}$ In this regard, the use of asymmetric organo- and metal-catalysis in fullerene chemistry has provided an easy access to optically active derivatives by precise stereocontrol of the newly formed asymmetric carbon atoms ${ }^{[8]}$ Conversely, control of the metal-

[] Dr. J. Marco-Martínez, S. Vidal, Dr. I. Fernández, Dr. S. Filippone, Prof. Dr. N. Martín

Departamento de Química Orgánica I, Facultad de Ciencias

Químicas, Universidad Complutense de Madrid

Ciudad Universitaria s/n, 28040 Madrid (Spain)

E-mail: salvatorefilippone@ucm.es nazmar@quim.ucm.es

Homepage: http://www.nazariomartingroup.com

Prof. Dr. N. Martín

IMDEA-Nanociencia, C/ Faraday, 9, Campus de la Universidad

Autónoma de Madrid 28049 Madrid (Spain)

(2) Supporting information and the ORCID identification number(s) for the author(s) of this article can be found under http://dx.doi.org/10. 1002/anie.201611475. centered chirality is not a trivial issue because of the configurational lability of the metals, which often undergo rapid ligand exchange. ${ }^{[4]}$

Herein, we report the first enantioselective synthesis of fullerene hybrids endowed with a stable stereogenic metal center whose configuration can be defined at will by the proper choice of chiral catalyst. In these reactions, iridium, rhodium, and ruthenium pyrrolidino[3,4:1,2][60]fullerene half-sandwich complexes with four new stereocenters are formed; namely the two $\mathrm{C} 2$ and $\mathrm{C} 5$ chiral carbon atoms at the pyrrolidine ring, the asymmetric nitrogen atom, and the transition-metal center (Scheme 1). The functionalization and
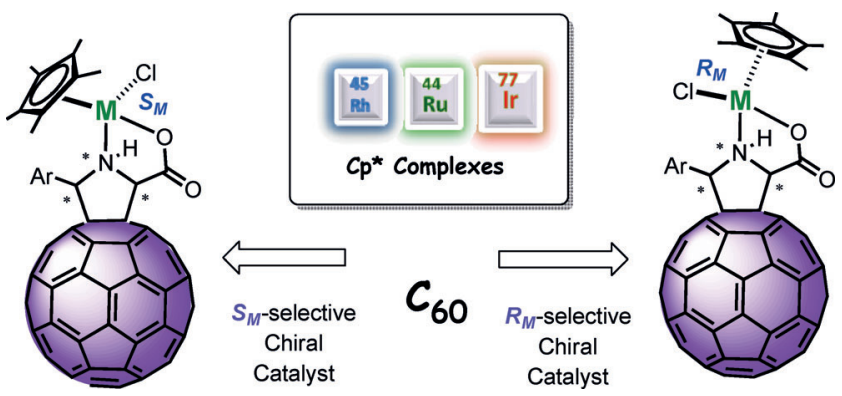

Scheme 1. Stereodivergent-at-metal synthesis of iridium, rhodium, and ruthenium half-sandwich [60]fullerene complexes.

stereochemistry of the chiral fullerene ligand determines the final configuration of the metal center. Interestingly, the chiral metal center does not undergo any epimerization process, as indicated by variable-temperature NMR analysis as well as by DFT theoretical calculations.

The syntheses of the chiral metal-fullerene hybrids were inspired by the preparation of half-sandwich complexes with aminocarboxylate ligands, ${ }^{[9]}$ and take advantage of the full control previously achieved in stereodivergent synthesis of pyrrolidino[60]fullerenes. ${ }^{[\mathrm{aa}, \mathrm{d}, \mathrm{e}]}$ Thus, the addition of a $t \mathrm{Bu}-\alpha-$ iminoester, endowed with a labile ester moiety for further hydrolysis and complexation, was directed enantioselectively toward each one of the four possible stereoisomeric pyrrolidino[60]fullerenes by selecting the appropriate chiral metal catalyst.

Thus, optically pure cis diastereomer $\mathbf{1}\left(\boldsymbol{S}_{C 2}, \boldsymbol{S}_{C 5}\right)$ was treated with pentamethylcyclopentadienyl $\left(\mathrm{Cp}^{*}\right)$ iridium dichloride dimer, $\left[\mathrm{Cp}^{*} \mathrm{IrCl}_{2}\right]_{2}$, in a one-pot procedure after ester hydrolysis with a trifluoroacetic acid/dichloromethane (TFA/DCM, 1:4) mixture, affording the iridium half-sandwich fullerene hybrid $\left(\boldsymbol{S}_{C 2}, \boldsymbol{S}_{C 5}, \boldsymbol{S}_{N}, \boldsymbol{S}_{\boldsymbol{I r}}\right)-\mathbf{1 I} \mathbf{r}^{[10]}$ in $77 \%$ yield (Scheme 2). As a result of the coordination of the pyrrolidino- 


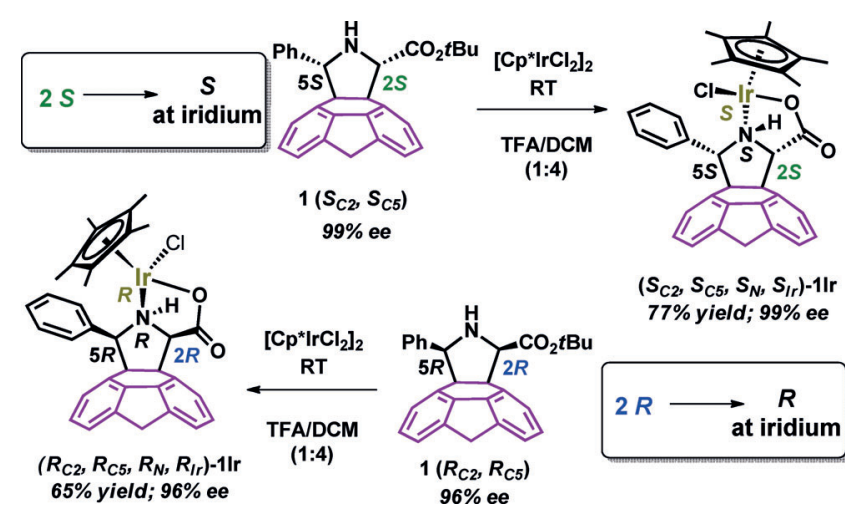

Scheme 2. Preparation of half-sandwich [60]fullerene complexes with a stable iridium-centered chirality.

[60]fullerene carboxylate moiety, two additional stereogenic centers are formed at the pyrrolidine nitrogen atom and at the iridium atom. As inferred from NOE experiments and in analogy with other related systems, ${ }^{[9]}$ the $\mathrm{N}-\mathrm{H}$ bond remains in a cis disposition with respect to the $\mathrm{C} 2-\mathrm{H}$ pyrrolidine bond; probably to avoid an unfavorable trans disposition between the two fused five-membered rings. Despite the fact two different configurations can be adopted by the iridium center, a single product is formed. This can be accounted for by the diastereospecific addition of the metal fragment, where its configuration is determined by the chirality of $\mathrm{C} 2$ of the pyrrolidine ring, without any loss of optical purity (Supporting Information). Thus, the configuration of the two new stereogenic centers is $S$, in agreement with the $S$ configuration of the $\mathrm{C} 2$ carbon atom. Moreover, in sharp contrast to related complexes based on a prolinate ligand, ${ }^{[8]}$ no evidence of formation of a diastereomeric mixture or epimerization process at the metal center has been found (see proceeding discussion). This behavior is very likely due to the presence of the aryl substituent on the pyrrolidine $\mathrm{C} 5$ carbon atom, that gives rise to a favorable interaction with the $\mathrm{Cp}^{*}$ group that prevents iridium epimerization. This positive interaction is known as a " $\beta$-phenyl effect" because of the aryl substitution at the beta-position of the metal. ${ }^{[1]}$ Furthermore, theoretical calculations underpin this experimental finding, revealing the occurrence of a stabilizing $\mathrm{C}-\mathrm{H} / \pi$ interaction between the hydrogen atoms of the pentamethylcyclopentadienyl moiety and the phenyl ring (see proceeding discussion).

As expected, we were also able to obtain the fullerene hybrids with the opposite $R$ configuration at the iridium metal atom using pyrrolidine $\mathbf{1}\left(\boldsymbol{R}_{\boldsymbol{C} 2}, \boldsymbol{R}_{C 5}\right)$ obtained from the catalytic system $\mathrm{Ag} /(R)$-BPE $\quad((R)$-BPE: (-)-1,2-bis$((2 R, 5 R)-2,5$-diphenylphospholano)ethane). Once again, the formed $\left(\boldsymbol{R}_{\boldsymbol{C} 2}, \boldsymbol{R}_{\boldsymbol{C} 5}, \boldsymbol{R}_{\boldsymbol{N}}, \boldsymbol{R}_{\boldsymbol{I}}\right)$-1Ir complex did not undergo any measurable loss of enantiomeric excess or an epimerization process.

Thus, each trans stereoisomer $\mathbf{2}\left(\boldsymbol{S}_{C 2}, \boldsymbol{R}_{C 5}\right)$ and $\mathbf{2}\left(\boldsymbol{R}_{C 2}, \boldsymbol{S}_{C 5}\right)$ (obtained using $\mathrm{Cu}^{\mathrm{II}} /(R)$ and $(S)$-DTBM-SEGPHOS, respectively; Supporting Information), was treated with [Cp* $\left.\mathrm{IrCl}_{2}\right]_{2}$ after the ester hydrolysis, affording the respective stereoisomers $\left(\boldsymbol{S}_{C 2}, \boldsymbol{R}_{C 5}, \boldsymbol{S}_{N}, \boldsymbol{S}_{I r}\right)$-2Ir and $\left(\boldsymbol{R}_{C 2}, \boldsymbol{S}_{C 5}, \boldsymbol{R}_{N}, \boldsymbol{R}_{I r}\right)$-2Ir exclusively (Scheme 3 ). Both compounds are the epimers at

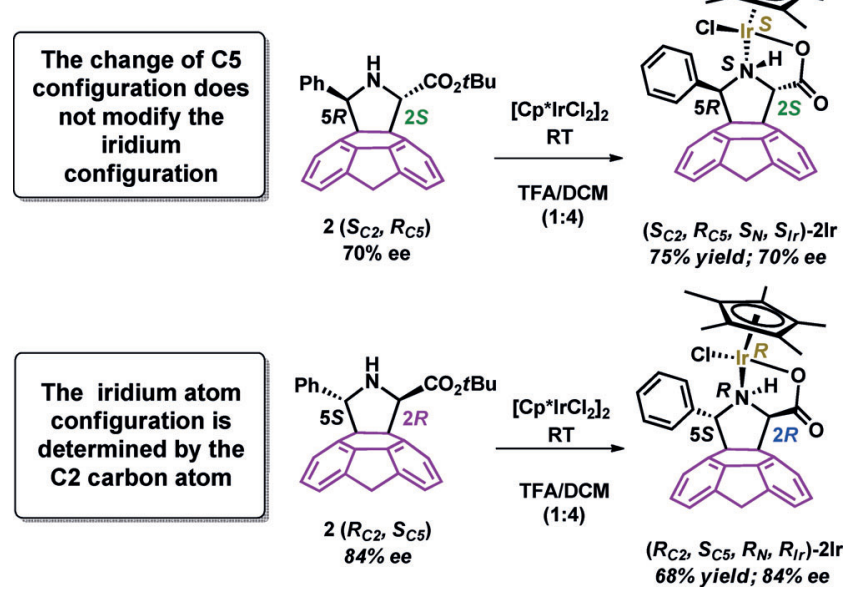

Scheme 3. Preparation of half-sandwich [60]fullerene complexes from the stereoselective trans pyrrolidino[60]fullerene isomers.

C5 of the aforementioned $\left(S_{C 2}, S_{C 5}, S_{N}, S_{I r}\right)$-1Ir and $\left(R_{C 2}, R_{C 5}\right.$, $\left.\boldsymbol{R}_{N}, \boldsymbol{R}_{I r}\right)$-1Ir isomers, while the remaining three stereogenic centers remain unchanged; the iridium metal atom maintains the same configuration as the $\mathrm{C} 2$ pyrrolidine carbon. Once again, it is worth noting the high stability of the stereogenic metal center. Actually, none of the four stereoisomers undergo an epimerization process in a range of temperatures from $-30^{\circ} \mathrm{C}$ to $90^{\circ} \mathrm{C}$, as determined by variable-temperature ${ }^{1} \mathrm{H}$ NMR spectroscopy and a determination of the enantiomeric excesses, which remained unaltered.

The aforementioned synthetic method was also compatible with other transition metals, such as rhodium and ruthenium, affording chiral hybrids with complete stereocontrol (Figure 1). While rhodium complexes, $\mathbf{1 R h}$, have been
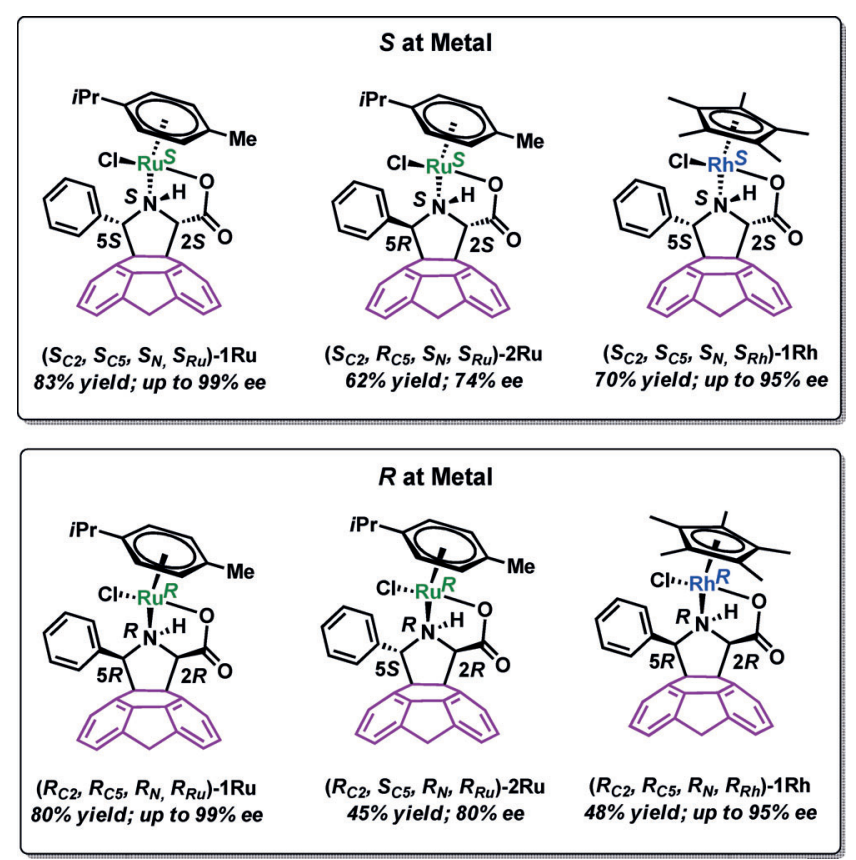

Figure 1. Ruthenium- and rhodium-based fullerene hybrids with $R$ or $S$ configuration at the metal center. 
isolated only from the cis pyrrolidine, Ru-pyrrolidino[60]fullerene hybrids $1 R \mathbf{u}$ and $2 R \mathbf{u}$, endowed with a $p$-cymene group, were obtained in good yields (45-83\%) and excellent stereoselectivities after reaction with $\left[\left(\eta^{6}-\right.\right.$ cymene) $\left.\mathrm{RuCl}_{2}\right]_{2}$ (Figure 1).

DFT calculations at the dispersion corrected RI-BP86D3/def2-SVP level ${ }^{[12]}$ were carried out to gain more insight into the configurational stability of the metal centers and the observed stereochemical outcome. To this end, we first focused on the two possible stereoisomers of $\mathbf{1} \mathbf{I r}$, namely the observed $\left(\boldsymbol{S}_{C 2}, \boldsymbol{S}_{C 5}, \boldsymbol{S}_{\boldsymbol{N}}, \boldsymbol{S}_{I r}\right)$-1Ir species and its transition metal epimer $\left(S_{C 2}, S_{C 5}, S_{N}, R_{I r}\right)$-1Ir.

Our calculations indicate that the former isomer is $8.7 \mathrm{kcal} \mathrm{mol}^{-1}$ more stable than the latter (Figure 2, top). A similar result was found when considering the strongly related 1Ru-isomers. In this case, the solely formed $\left(\boldsymbol{S}_{C 2}, \boldsymbol{S}_{C 5}, \boldsymbol{S}_{N}, \boldsymbol{S}_{R u}\right)$ 1Ru compound was computed to be $14.5 \mathrm{kcalmol}^{-1}$ more stable than $\left(\boldsymbol{S}_{\boldsymbol{C} 2}, \boldsymbol{S}_{\boldsymbol{C} 5}, \boldsymbol{S}_{\boldsymbol{N}}, \boldsymbol{R}_{\boldsymbol{R} u}\right)$-1Ru (Figure 2, bottom). Both results are fully consistent with the observed diastereoselectivity and with the aforementioned lack of epimerization at the transition-metal fragment.

Two main reasons are responsible for the much higher computed stability of $\left(S_{C 2}, S_{C 5}, S_{N}, S_{I r}\right)-1 \operatorname{Ir}$ or $\left(S_{C 2}, S_{C 5}, S_{N}\right.$, $\left.S_{R u}\right)$-1Ru stereoisomers. On one hand, the less stable isomers

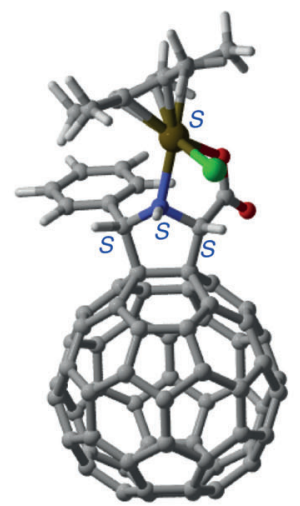

$\left(s_{C 2}, s_{C 5}, s_{N}, s_{I r}\right)-1 / r$ $E_{\text {rel }}=0.0 \mathrm{kcal} / \mathrm{mol}$

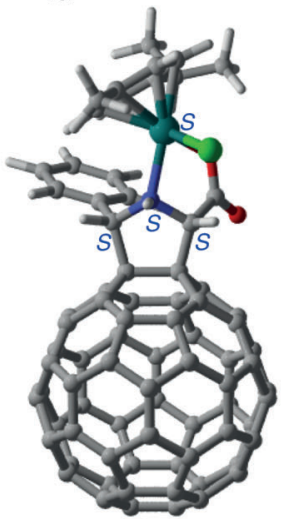

$\left(S_{C 2}, s_{C 5}, s_{N}, s_{R u}\right)-1 R u$ $E_{\text {rel }}=0.0 \mathrm{kcal} / \mathrm{mol}$

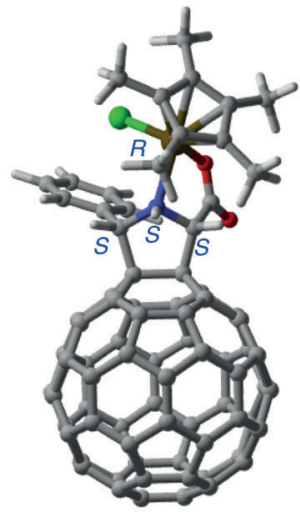

$\left(S_{C 2}, S_{C 5}, S_{N}, R_{l r}\right)-1 / r$ $E_{\text {rel }}=8.7 \mathrm{kcal} / \mathrm{mol}$

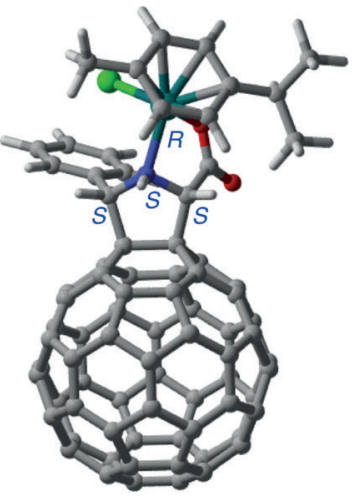

$\left(S_{C 2}, S_{C 5}, S_{N}, R_{R u}\right)-1 R u$ $E_{\text {rel }}=14.5 \mathrm{kcal} / \mathrm{mol}$
Figure 2. Fully optimized geometries of the possible stereoisomers of IIr (top) and $\mathbf{1 R u}$ (bottom). All data have been computed at the RI-BP86-D3/def2-SVP level. are affected by a strong repulsive interaction between the lone pair of the chloride ligand and the $\pi$-electrons of the adjacent phenyl ring, which is not present in the $\boldsymbol{S}_{\boldsymbol{I r}}$ or $\boldsymbol{S}_{\boldsymbol{R} u}$ isomers. Indeed, the computed $\mathrm{Cl} \cdots \pi$-centroid distances of $3.35 \AA$ and $3.38 \AA$ for $\boldsymbol{R}_{\boldsymbol{I r}}$ and $\boldsymbol{R}_{\boldsymbol{R} u}$, respectively, are shorter than the sum of the van der Waals radii of the involved atoms $(\mathrm{Cl}=1.75 \AA$, phenyl group $=1.7 \AA)$.

On the other hand, there is a stabilizing $\mathrm{CH}-\pi$ interaction established between two methyl groups of the $\mathrm{Cp}^{*}$ ligand and the adjacent phenyl substituent at C5 in the more stable $\left(\boldsymbol{S}_{C 2}\right.$, $\left.\boldsymbol{S}_{C 5}, \boldsymbol{S}_{N}, \boldsymbol{S}_{I r}\right)$-1Ir isomer. The computed shortest $\mathrm{CH} \cdots \pi$ centroid distance of $2.54 \AA$ fits nicely in the typical range reported for this type of non-covalent interaction. ${ }^{[13]} \mathrm{A}$ similar $\mathrm{CH}-\pi$ interaction is found in the analogous $\left(\boldsymbol{S}_{C 2}, \boldsymbol{S}_{C 5}, \boldsymbol{S}_{N}, \boldsymbol{R}_{\boldsymbol{R} u}\right)$ 1Ru species. The occurrence of this stabilizing interaction (that is, the $\beta$-phenyl effect described in the aforementioned text), which can only occur in the $S$-metal isomers, can be easily visualized by means of the NCIPLOT method. ${ }^{[14]}$ As clearly shown in Figure $3 \mathrm{a}$, there is a non-covalent attractive interaction (green surface) between the methyl groups of the $\mathrm{Cp}^{*}$ ligand and the adjacent phenyl ring. The presence of these $\mathrm{CH} \cdots \pi$ interactions is also confirmed by the AIM method, ${ }^{[15]}$ which locates two bond-critical points associated with bond paths running between the involved hydrogen and carbon atoms (Figure $3 \mathrm{~b}$ ). Therefore, the combination of these lone pair $(\mathrm{Cl})-\pi$ and $\mathrm{CH}-\pi$ interactions is partially responsible for the computed energy differences between the considered stereoisomers.
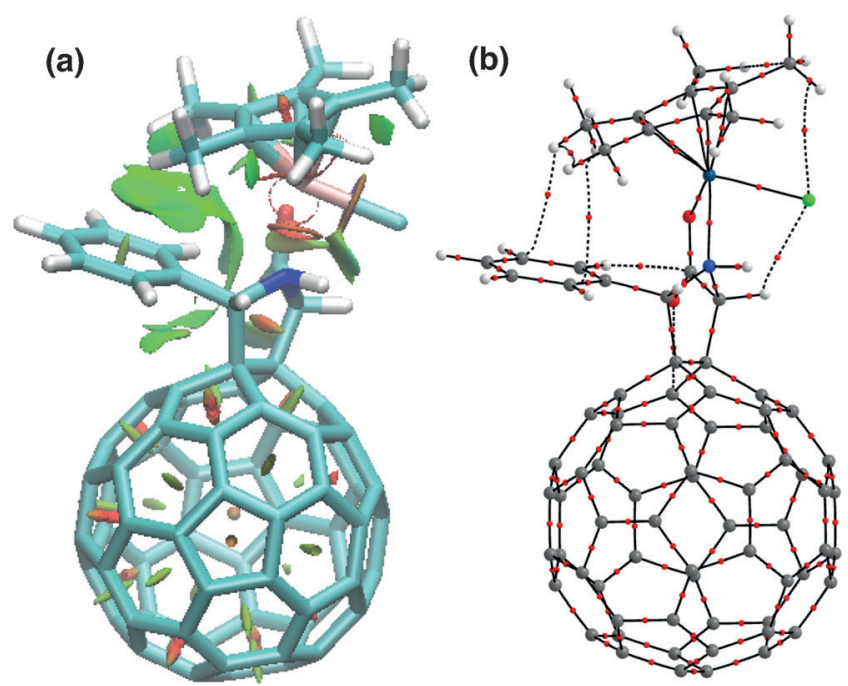

Figure 3. a) Representation of non-covalent interactions in $\left(S_{C 2}, S_{C 5}\right.$, $\left.S_{N}, S_{I r}\right)$-1 lr. b) AIM diagram for $\left(S_{C 2}, S_{C 5}, S_{N}, S_{I r}\right)$-1lr. The lines connecting the nuclei are the bond paths, while the small red spheres indicate the corresponding bond critical points.

In summary, we have carried out straightforward syntheses of a new family of chiral fullerene-metal hybrids for the first time-namely, iridium, rhodium, and ruthenium pyrrol idino[3,4:1,2][60]fullerene half-sandwich complexes-with complete control over the four new stereogenic centers, including the metal atom. 
Stereodivergent-at-metal synthesis is based on enantioselective synthesis of suitably functionalized pyrrolidino[60]fullerenes and further diastereoselective addition of metal complexes, leading to half-sandwich complexes with fulleroaminocarboxylate chiral ligands. Interestingly, the stereochemical outcome of the four chiral centers stems from the chiral catalysts employed and the diastereoselective addition of the metal complexes used (iridium, rhodium, or ruthenium). The observed lack of epimerization processes in the metal complexes has been rationalized by means of DFT calculations, which reveal the presence of a repulsive interaction between the lone pair of the chloride ligand and the $\pi$-electrons of the adjacent phenyl ring, as well as a stabilizing $\mathrm{CH}-\pi$ interaction ( $\beta$-phenyl effect) between two methyl groups of the $\mathrm{Cp}^{*}$ ligand and the adjacent phenyl substituent at $\mathrm{C} 5$ occurring in the $S$-metal isomers.

Stereodivergent control in fullerene derivatives endowed with a chiral metal atom represents new progress in facile preparative procedures for chiral fullerenes. The findings pave the way toward application of these new fullerene-metal hybrid systems, which are of interest in their own right but are also potential catalysts for a variety of (stereo)chemical reactions.

\section{Acknowledgements}

Financial support by the European Research Council (ERC320441-Chirallcarbon), the Ministerio de Economía y Competitividad (MINECO) of Spain (project CTQ2014-52045-R) and the Comunidad Autónoma de Madrid (PHOTOCARBON project S2013/MIT-2841) is acknowledged I.F. is grateful for financial support from the Spanish MINECO-FEDER (Grants CTQ2013-44303-P and CTQ2014-51912-REDC), and Fundación BBVA (Convocatoria 2015 de Ayudas Fundación BBVA a Investigadores $y$ Creadores Culturales).

\section{Conflict of interest}

The authors declare no conflict of interest.

Keywords: chiral metallofullerenes - chiral-at-metal complexes · enantioselective synthesis - fullerene chemistry
[1] W. Krätschmer, L. D. Lamb, K. Fostiropoulos, D. R. Huffman, Nature 1990, 347, 354-358.

[2] A. L. Balch, M. M. Olmstead, Chem. Rev. 1998, 98, 2123-2166.

[3] M. A. Lebedeva, T. W. Chamberlain, A. N. Khlobystov, Chem. Rev. 2015, 115, 11301-11351.

[4] a) E. B. Bauer, Chem. Soc. Rev. 2012, 41, 3153-3167; b) C. Tian, L. Gong, E. Meggers, Chem. Commun. 2016, 52, 4207-4210; c) C. Ganter, Chem. Soc. Rev. 2003, 32, 130-138; d) H. Brunner, Angew. Chem. Int. Ed. 1999, 38, 1194-1208; Angew. Chem. 1999, 111, 1248-1263.

[5] W.-Y. Yeh, J. Organomet. Chem. 2015, 784, 13-23.

[6] Y.-W. Zhong, Y. Matsuo, E. Nakamura, Chem. Asian J. 2007, 2, $358-366$.

[7] a) V. V. Bashilov, P. V. Petrovskii, V. L. Sokolov, F. M. Dotgushin, A. L. Yanovsky, Y. T. Struchkov, Russ. Chem. Bull. 1996, 45, 1207-1213; b) V. I. Sokolov, V. V. Bashilov, F. M. Dolgushin, N. V. Abramova, K. K. Babievsky, A. G. Ginzburg, P. V. Petrovskii, Tetrahedron Lett. 2009, 50, 5347-5350; c) V. V. Bashilov, P. V. Petrovskii, V. I. Sokolov, Russ. Chem. Bull. 1993, 42, $392-$ 393.

[8] a) S. Filippone, E. E. Maroto, A. Martín-Domenech, M. Suárez, N. Martín, Nat. Chem. 2009, 1, 578-582; b) J. Marco-Martínez, V. Marcos, S. Reboredo, S. Filippone, N. Martín, Angew. Chem. Int. Ed. 2013, 52, 5115-5119; Angew. Chem. 2013, 125, 52195223 ; c) J. Marco-Martínez, S. Reboredo, M. Izquierdo, V. Marcos, J. L. López, S. Filippone, N. Martín, J. Am. Chem. Soc. 2014, 136, 2897-2904; d) E. E. Maroto, S. Filippone, A. MartínDomenech, M. Suárez, N. Martín, J. Am. Chem. Soc. 2012, 134, 12936-12938; e) E. E. Maroto, S. Filippone, M. Suárez, R. Martínez-Álvarez, A. de Cózar, F. P. Cossío, N. Martín, J. Am. Chem. Soc. 2014, 136, 705 -712; f) E. E. Maroto, M. Izquierdo, S. Reboredo, J. Marco-Martínez, S. Filippone, N. Martín, Acc. Chem. Res. 2014, 47, 2660-2670.

[9] D. Carmona, M. Pilar Lamata, F. Viguri, E. San José, A. Mendoza, F. J. Lahoz, P. García-Orduña, R. Atencio, L. A. Oro, J. Organomet. Chem. 2012, 717, 152-163.

[10] The absolute configurations of the stereogenic metal centers have been determined by following the Cahn-Ingold-Prelog rules and assigning the highest priority to the hapto ligands, as indicated in the literature. See reference [4a].

[11] H. Brunner, Angew. Chem. Int. Ed. Engl. 1983, 22, 897-907; Angew. Chem. 1983, 95, 921-931.

[12] For computational details see the Supporting Information.

[13] M. Nishio, M. Hirota, Y. Umezawa, The $\mathrm{CH} / \pi$ Interaction: Evidence, Nature and Consequences, Wiley, New York, 1998.

[14] E. R. Johnson, S. Keinan, P. Mori-Sánchez, J. Contreras-García, A. J. Cohen, W. Yang, J. Am. Chem. Soc. 2010, 132, 6498-6506.

[15] R. F. W. Bader, Atoms in Molecules: A Quantum Theory, Clarendon Press, Oxford, 1994.

Manuscript received: November 23, 2016

Final Article published: 


\section{Communications}

Fullerene Chemistry

J. Marco-Martínez, S. Vidal, I. Fernández, S. Filippone,* N. Martín* III-III

Stereodivergent-at-Metal Synthesis of [60]Fullerene Hybrids

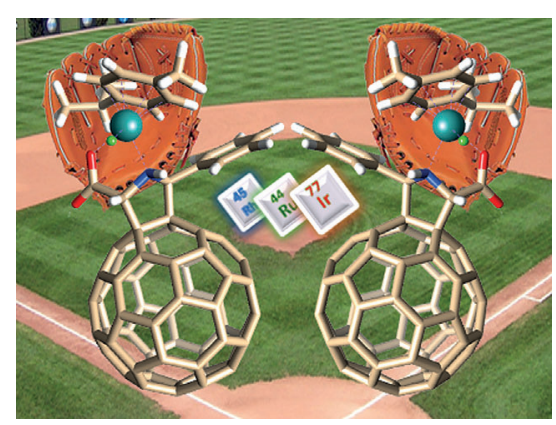

Chirality at the metal: Fullerene hybrids with iridium-, rhodium-, or rutheniumcentered chirality were prepared by precise control of four newly formed stereogenic centers. The resulting complexes are configurationally stable and do not undergo an epimerization process. 